\title{
Statistical Mechanics of Lattice Systems
}

\author{
G. Gallavottit and S. Mrracle-Sole*
}

Institut des Hautes Etudes Scientifiques

91. Bures-sur-Yvette - France

Received March 25, 1967

Abstract. We study the thermodynamic limit for a classical system of particles on a lattice and prove the existence of infinite volume correlation functions for a "large" set of potentials and temperatures.

\section{$\S 1$. Introduction and Notations}

In this article we shall study the statistical mechanics of a classical system on a $\boldsymbol{\nu}$-dimensional lattice $Z^{\nu}$. We assume that at each lattice point there can be either 0 or 1 particle. We suppose that the particles interact through symmetric translationally invariant many body potentials $\Phi^{(k)}\left(x_{1} \ldots x_{k}\right)$. Let $X=\left\{x_{1}, \ldots, x_{N}\right\}$ be a finite subset of $Z^{v}$, then the potential energy $U$ of $N$ particles located at $x_{1}, x_{2}, \ldots, x_{N}$ is:

$$
U(X)=\sum_{k=1}^{\infty} \frac{1}{k !} \sum_{\left(i_{1}, \ldots, i_{k}\right) \in\{1, \ldots, N\}}^{\neq} \Phi^{(k)}\left(x_{i_{1}}, \ldots, x_{i_{k}}\right)
$$

where $\stackrel{\neq}{\sum}$ extends over all $k$-ples $i_{1}, \ldots, i_{k}$ of distinct indices (between 1 and $N)$; in particular $U(\emptyset)=0$. We shall consider only interactions $\Phi=\left(\Phi^{(k)}\right)_{k \geqq 1}$ such that

$$
\|\Phi\|=\sum_{k=1}^{\infty} \frac{1}{k !} \sum_{0 \neq x_{1} \ldots x_{k-1} \in Z^{v}}^{\neq}\left|\Phi^{(k)}\left(0, x_{1}, \ldots, x_{k-1}\right)\right|<+\infty
$$

where the second sum extends over all $(k-1)$-ples $x_{1}, \ldots, x_{k-1}$ of distinct lattice points different from the origin 0 of $Z^{v}$. With respect to the norm (2) the set $\mathscr{B}$ of interactions $\Phi$ such that $\|\Phi\|<+\infty$ is a (real) Banach space.

\section{§ 2. Definitions and Inequalities}

From (1) and (2) we deduce the following stability property:

$$
\left|U\left(\left\{x_{1}, \ldots, x_{N}\right\}\right)\right| \leqq N\|\Phi\| \text {. }
$$

We define a subspace $\mathscr{B}^{\prime}$ of $\mathscr{B}$ by

$$
\mathscr{B}^{\prime}=\left\{\Phi \in \mathscr{B}: \Phi^{(1)}=0\right\}
$$

We may write $\Phi=\left(-\mu, \Phi^{\prime}\right)$ for every $\Phi \in \mathscr{B}$ with $\mu=-\Phi^{(1)}$ and $\Phi^{\prime} \in \mathscr{B}$. We interpret $\mu$ as chemical potential and denote by $U^{\prime}$ the potential energy corresponding to $\Phi^{\prime}$.

* On partial leave from the University of Aix-Marseille.

22 Commun. math. Phys., Vol. 5 
If $\beta$ is the inverse temperature, the grand partition function corresponding to the region (finite set) $\Lambda$ is then, if $N(X)$ is the number of points of $X$ :

$$
\Xi\left(\beta, \mu, \Phi^{\prime}\right)=\sum_{X \leqq \Lambda} e^{-\beta U(X)}=\sum_{X \leqq \Lambda} e^{\beta \mu N(X)} e^{-\beta U^{\prime}(X)} .
$$

It is notationally convenient to define

$$
Z_{\Lambda}(\Phi)=\sum_{X \leqq \Lambda} e^{-U(X)}
$$

and, if $V(\Lambda)$ is the number of points of $\Lambda$ :

Then

$$
P_{\Lambda}(\Phi)=V(\Lambda)^{-1} \log Z_{\Lambda}(\Phi) .
$$

$\Xi\left(\beta, \mu, \Phi^{\prime}\right)=Z_{\Lambda}\left(\beta\left(-\mu, \Phi^{\prime}\right)\right) ; \quad Z_{\Lambda}\left(\Phi^{(1)}, \Phi^{\prime}\right)=\Xi\left(1,-\Phi^{(1)}, \Phi^{\prime}\right)$.

Proposition 1. If $\Phi^{\prime}, \Psi^{\prime} \in \mathscr{B}^{\prime}$ then

$$
Z_{\Lambda}\left(\Phi^{(1)}+\left\|\Psi^{\prime}\right\|, \Phi^{\prime}\right) \leqq Z_{\Lambda}\left(\Phi^{(1)}, \Phi^{\prime}+\Psi^{\prime}\right) \leqq Z_{\Lambda}\left(\Phi^{(1)}-\left\|\Psi^{\prime}\right\|, \Phi^{\prime}\right) .
$$

Let indeed $V^{\prime}$ be the potential energy corresponding to $\Psi^{\prime}$ then

$$
\begin{gathered}
\left(\Phi^{(1)}-\left\|\Phi^{\prime}\right\|\right) N(X)+U^{\prime}(X) \leqq \Phi^{(1)} N(X)+U^{\prime}(X)+V^{\prime}(X) \leqq \\
\leqq\left(\Phi^{(1)}+\|\Psi\|\right) N(X)+U^{\prime}(X) .
\end{gathered}
$$

Where $N(X)$ is the number of points of $X$. The result then follows taking the exponentials and summing over $X$.

Proposition 2. If $\Phi \in \mathscr{B}$,

$$
\log \left(1+e^{-\Phi(1)}-\left\|\Phi^{\prime}\right\|\right) \leqq P_{\Lambda}(\Phi) \leqq \log \left(1+e^{-\Phi^{(1)}+\left\|\Phi^{\prime}\right\|}\right) .
$$

This is obtained from proposition 1 by taking $\Phi^{\prime}=0$.

Proposition 3. The function $\Phi \rightarrow P_{\Lambda}(\Phi)$ is convex on $\mathscr{B}$.

The proof is standard ${ }^{1}$ and will be omitted.

Proposition 4. If $\Phi^{\prime} \in \mathscr{B}^{\prime}$, the following inequality holds:

$$
\left|P_{\Lambda}\left(\Phi^{(1)}-\lambda, \Phi^{\prime}\right)-P_{\Lambda}\left(\Phi^{(1)}+\lambda, \Phi^{\prime}\right)\right| \leqq 2 \lambda \quad \forall \Phi^{(1)} \in R, \forall \lambda \geqq 0 .(9)
$$

This follows from the fact that the derivative of $P_{\Lambda}\left(\Phi^{(1)}, \Phi^{\prime}\right)$ with respect to $\Phi^{(1)}$ is the expectation value of $[-N(X) / V(\Lambda)]$ :

$$
\frac{d P_{\Lambda}\left(\Phi^{(1)}, \Phi^{\prime}\right)}{d \Phi^{(1)}}=-\frac{\sum_{X \subseteq \Lambda} e^{-\delta(X)} N(X)}{Z_{\Lambda}(\Phi) V(\Lambda)}
$$

and is therefore contained in the interval $[-1,0]$.

\section{§ 3. Existence and Properties of the Thermodynamic Limit}

Let $\mathscr{B}_{0} \subset \mathscr{B}$ consist of those $\Phi$ which have finite range i. e. $\Phi^{(k)} \equiv 0$ for $k$ sufficiently large and $\Phi^{(k)}\left(0, x_{1}, \ldots, x_{k-1}\right)$ vanishes except for a finite number of values of $x_{1}, \ldots, x_{k-1}$.

${ }_{1}$ It follows from the convexity criteria for many variables functions and the Schwartz inequality. 
Proposition 5. If $\Phi \in \mathscr{B}_{0}$, the following limit exists

$$
P(\Phi)=\lim _{\Lambda \rightarrow \infty} P_{\Lambda}(\Phi)
$$

This result is well known (see [1], [2], [3], [4], [5]).

In this proposition and in the following $\Lambda$ may be taken a parallelopiped and $\Lambda \rightarrow \infty$ means that each side of $\Lambda$ tends to $\infty$. It is also possible to let $\Lambda$ to go to $\infty$ in a more general manner (see [6], [7] for a definition of Van-Hove convergence to $\infty$ ).

Theorem 1. If $\Phi \in \mathscr{B}$, the following limit exists

$$
P(\Phi)=\lim _{\Lambda \rightarrow \infty} P_{\Lambda}(\Phi)
$$

and satisfies the following properties

i) if $\Phi^{\prime}, \Psi^{\prime} \in \mathscr{B}^{\prime}$ then

$$
P\left(\Phi^{(1)}+\left\|\Psi^{\prime}\right\|, \Phi^{\prime}\right) \leqq P\left(\Phi^{(1)}, \Phi^{\prime}+\Psi^{\prime}\right) \leqq P\left(\Phi^{(1)}-\left\|\Psi^{\prime}\right\|, \Phi^{\prime}\right) ;
$$

ii) $\log \left(1+e^{-\Phi(1)-\left\|\Phi^{\prime}\right\|}\right) \leqq P(\Phi) \leqq \log \left(1+e^{-\Phi(1)}+\left\|\Phi^{\prime}\right\|\right)$;

iii) the functional $P(\cdot)$ is convex and continuous on the Banach space $\mathscr{B}$.

Let $\Phi_{n}^{\prime} \in \mathscr{B}_{0}$ be such that $\lim _{n \rightarrow \infty}\left\|\Phi_{n}^{\prime}-\Phi^{\prime}\right\|=0$. From proposition 1 we obtain:

$$
P_{\Lambda}\left(\Phi^{(1)}+\left\|\Phi^{\prime}-\Phi_{n}^{\prime}\right\|, \Phi_{n}^{\prime}\right) \leqq P_{\Lambda}(\Phi) \leqq P_{\Lambda}\left(\Phi^{(1)}-\left\|\Phi^{\prime}-\Phi_{n}^{\prime}\right\|, \Phi_{n}^{\prime}\right)
$$

On the other hand $Z_{\Lambda}\left(\Phi^{(1)}, \Phi^{\prime}\right)$ is a decreasing function of $\Phi^{(1)}$ so that: $P_{\Lambda}\left(\Phi^{(1)}+\left\|\Phi^{\prime}-\Phi_{n}^{\prime}\right\|, \Phi_{n}^{\prime}\right) \leqq P_{\Lambda}\left(\Phi^{(1)}, \Phi_{n}^{\prime}\right) \leqq P_{\Lambda}\left(\Phi^{(1)}-\left\|\Phi^{\prime}-\Phi_{n}^{\prime}\right\|, \Phi_{n}^{\prime}\right)$

from proposition 4 , the difference between extreme terms in (14) and (15) is bounded by $2\left\|\Phi^{\prime}-\Phi_{n}^{\prime}\right\|$, hence

$$
\left|P_{\Lambda}(\Phi)-P_{\Lambda}\left(\Phi^{(1)}, \Phi_{n}^{\prime}\right)\right| \leqq 2\left\|\Phi^{\prime}-\Phi_{n}^{\prime}\right\|
$$

from this it follows that

$$
\lim _{n \rightarrow \infty} P_{\Lambda}\left(\Phi^{(1)}, \Phi_{n}^{\prime}\right)=P_{\Lambda}(\Phi)
$$

uniformly in $\Lambda$. On the other hand by proposition 5

$$
\lim _{\Lambda \rightarrow \infty} P_{\Lambda}\left(\Phi^{(1)}, \Phi_{n}^{\prime}\right)=P\left(\Phi^{(1)}, \Phi_{n}^{\prime}\right) .
$$

The existence of the limits (17) and (18) and the uniformity of (17) imply the existence of

$$
\lim _{\Lambda \rightarrow \infty} P_{\Lambda}(\Phi)=\lim _{\Lambda \rightarrow \infty} \lim _{n \rightarrow \infty} P_{\Lambda}\left(\Phi^{(1)}, \Phi_{n}^{\prime}\right) .
$$

This proves (11), (i) follows then from proposition 1; (ii) from proposition 2 ; the convexity of $P(\cdot)$ implies its continuity in $\Phi^{(1)}$ and then by (i) its continuity in $\Phi$ follows. 
Remark. From the above theorem we have the existence of

$$
\beta p\left(\beta, \mu, \Phi^{\prime}\right)=\lim _{\Lambda \rightarrow \infty} V(\Lambda)^{-1} \log \Xi\left(\beta, \mu, \Phi^{\prime}\right)
$$

where $p$ is the thermodynamique pressure:

$$
p\left(\beta, \mu, \Phi^{\prime}\right)=\beta^{-1} P\left(\beta\left(-\mu, \Phi^{\prime}\right)\right) .
$$

\section{$\$ 4$. Existence of Correlation Functions}

Let $\Lambda$ be a finite subset of $Z^{v}, \Phi \in \mathscr{B}$, the correlation function is defined by:

$$
\varrho_{\Phi}(X)=Z_{\Lambda}(\Phi)^{-1} \sum_{\substack{Y \subseteq \Lambda \\ Y \cap X=\emptyset}} e^{-U(X \cup Y)}
$$

if $X \leqq \Lambda$ and $\varrho_{\Phi}(X)=0$ otherwise. By averaging over translations we get

$$
\bar{\varrho}_{\Phi}\left(\left\{x_{1} \ldots x_{n}\right\}\right)=V(\Lambda)^{-1} \sum_{X \in Z^{\nu}} \varrho_{\Phi_{A}}\left(x_{1}+x, \ldots, x_{n}+x\right)
$$

so that if $\Psi \in \mathscr{B}$ with corresponding potential energy $V$ :

$$
\begin{aligned}
& \sum_{n \geqq 1} \frac{1}{n !} \sum_{0 \neq x_{2} \ldots x_{n} \in Z^{\nu}} \bar{\varrho}_{\Phi \Lambda}\left(0, x_{2} \ldots x_{n}\right) \Phi^{(n)}\left(0, x_{\mathbf{2}} \ldots x_{n}\right) \\
& =V(\Lambda)^{-1} \sum_{X \neq \varnothing} \varrho_{\Phi \Lambda}(X) \Psi(X)=Z_{\Lambda}(\Phi)^{-1} V(\Lambda)^{-1} \sum_{\substack{\Phi \neq X ; Y \subseteq \Lambda \\
X \cap Y=\emptyset}} e^{-U(X \cup Y)} \Psi(X) \\
& =Z_{\Lambda}(\Phi)^{-1} V(\Lambda)^{-1} \sum_{X \sqsubseteq \Lambda} e^{-U(X)} V(X) .
\end{aligned}
$$

Let $T \subset \mathscr{B}$ be the set of $\Phi$ such that the graph of $P$ has a unique tangent plane at $\Phi$, i. e. there exists a unique $\alpha_{\Phi}$ in the dual $\mathscr{B}^{*}$ of $\mathscr{B}$ such that for all $\Psi \in \mathscr{B}$

$$
P(\Phi+\Psi) \geqq P(\Phi)-\alpha_{\Phi}(\Psi)
$$

we note that $\alpha_{\Phi}(\Psi)$ can be interpreted as the functional derivative of $P(\Phi)$ in the direction $\Psi[8]$.

Theorem 2. If $\Phi \in T$ then if $V$ is the potential energy associated with any $\Psi \in \mathscr{B}$ the limit

$$
\lim _{\Lambda \rightarrow \infty} Z_{\Lambda}(\Phi)^{-1} V(\Lambda)^{-1} \sum_{X \sqsubseteq \Lambda} e^{-U(X)} V(X)=\alpha_{\Phi}(\Psi)
$$

exists and defines an element $\alpha_{\Phi} \in \mathscr{B}^{*}$; the following limit therefore exists:

$$
\lim _{\Lambda \rightarrow \infty} \bar{\varrho}_{\Phi, \Lambda}(X)=\bar{\varrho}_{\Phi}(X)
$$

and defines the infinite volume correlation function $\bar{\varrho}_{\Phi}$.

For finite $\Lambda$, the function $P_{\Lambda}(\cdot)$ has a unique tangent plane at any $\Phi \in \mathscr{B}$ corresponding to $\alpha_{\Phi_{A}} \in \mathscr{B}^{*}$ :

$$
\alpha_{\Phi, \Lambda}(\Psi)=Z_{\Lambda}(\Phi)^{-1} V(\Lambda)^{-1} \sum_{X \sqsubseteq \Lambda} e^{-U(X)} V(X)
$$


From (3) it is clear that $\left|\alpha_{\Phi}^{\left(\Psi_{\Lambda}\right)}\right| \leqq\|\Psi\|$, i. e. $\left\|\alpha_{\Phi \Lambda}\right\| \leqq 1$. Let $A$ be a total sequence in $\mathscr{B}$ ( $\mathscr{B}$ is separable), one can choose a sequence $\Lambda_{n} \rightarrow \infty$ as $n \rightarrow \infty$ such that $\alpha_{\Phi, \Lambda_{n}}(\Psi)$ converges for every $\Psi \in A$. Since $\left\|\alpha_{\Phi \Lambda_{n}}\right\| \leqq$ $\leqq 1, \alpha_{\Phi \Lambda_{n}}$ converges weakly.

Let $(\Phi+\Psi, \xi)$ be a point strictly above the graph of $P$ in $\mathscr{B} \times R$, then for large $\Lambda,(\Phi+\Psi, \xi)$ is above the graph of $P_{\Lambda}$ and therefore of $\alpha_{\Phi}$ : in particular if $\alpha_{\Phi}$ is the limit of $\alpha_{\Phi A_{n}}$

$$
\xi=P(\Phi)-\alpha_{\Phi}(\Psi)+\alpha_{\Phi}(\Phi)
$$

is the equation of a tangent plane to $P$ at $\Phi$. If $\Phi \in T$, the tangent plane is unique, therefore

$$
\underset{\Lambda \rightarrow \infty}{\text { weak }} \lim _{\Phi \Lambda} \alpha_{\Phi}=\alpha_{\Phi}
$$

Remark. If $-\left.\frac{d P(\Phi+\lambda \Psi)}{d \lambda}\right|_{\lambda=0}=\alpha_{\Phi}(\Psi)$ exists for a certain $\Psi$ then

$$
\lim _{\Lambda \rightarrow \infty} \alpha_{\Phi \Lambda}(\Psi)=\alpha_{\Phi}(\Psi) \text {. }
$$

We note also that the existence of $\left.\frac{d P(\Phi+\lambda \Psi)}{d \lambda}\right|_{\lambda=0}$ for $\Psi$ in a total set is a necessary and sufficient condition for the existence of a unique tangent plane at $\Phi$.

These results follow by inspection of the proof of the above theorem.

We conclude with the following:

Theorem 3. i) The set $T$ contains a countable intersection of dense open subsets of $\mathscr{B}$ and therefore is dense (Baire theorem [9]).

ii) There exists a dense subset $T^{\prime}$ of $\mathscr{B}^{\prime}$ such that for $\Phi^{\prime} \in T^{\prime}$ and almost every $(\beta, \mu) \in R_{+} \times R$ the point $\beta\left(-\mu, \Phi^{\prime}\right) \in T$.

i) follows by inspection of the proof of reference [10].

Let $e_{n}$ be a base of normalized vectors on the space $\mathscr{B}^{\prime}[11]$. Let $\Phi_{(0)}^{\prime}$ be an arbitrary point of $\mathscr{B}^{\prime}$ and let $\left\{C_{n}\right\}$ be a sequence of positive numbers such that $\sum_{n=1}^{\infty} C_{n}<+\infty$. Let $K$ be the set

$$
K=\left\{\Phi^{\prime} \in \mathscr{B}^{\prime}:\left|\Phi_{n}^{\prime}-\Phi_{(0) n}^{\prime}\right|<C_{n}\right\}
$$

where $\Phi_{n}^{\prime}, \Phi_{(0) n}^{\prime}$ are the components of $\Phi^{\prime}, \Phi_{(0)}^{\prime}$ along $e_{n}$.

Let us consider the space $R_{+} \times R \times K$ of the variables $\left(\beta, \Phi^{(1)}, \Phi^{\prime}\right)$ as a topological space with the topology product of the natural topologies on $R_{+}$and $R$ and the relative topology on $K$ as a subset of $\mathscr{B}^{\prime}$ (it is easy to see that this topology on $K$ is identical with the product topology on $K$ considered as $\prod_{n=1}^{\infty} I_{n}$ where $\left.I_{n}=\left(-C_{n},+C_{n}\right)\right)$.

Let us introduce on $R_{+} \times R$ a normalized measure $g\left(d \beta d \Phi^{(1)}\right)$ equivalent to the Lebesgue measure and on $K$ the measure $\gamma\left(d \Phi^{\prime}\right)$ $=\prod_{n=1}^{\infty} \frac{d \Phi_{n}^{\prime}}{2 C_{n}}$. Let $\mu=g \times \gamma$ be the product measure of $g$ and $\gamma$ defined 
on (the Borel sets of) $R_{+} \times R \times K$. It is convenient to introduce the vector $e_{0}=(1,0) \in \mathscr{B}$.

Now the set $B_{n}$ of points $\left(\beta, \Phi^{(1)}, \Phi^{\prime}\right) \in R_{+} \times R \times K$ where the derivative $\frac{d}{d \lambda} P\left(\beta\left(\Phi+\lambda e_{n}\right)\right)$ does not exist is a Borel set of $R_{+} \times R \times K$ since

$$
B_{n}=\bigcup_{k=1}^{\infty} \bigcap_{N=1}^{\infty} \bigcup_{|s|,|t|>N} C_{k s t}^{n}, \quad n=0,1, \ldots
$$

where $k, N$ are positive integers and $s, t$ are integers and

$$
\begin{aligned}
C_{k s t}^{n}=\left\{\left(\beta, \Phi^{(1)}, \Phi^{\prime}\right) \in R_{+} \times R\right. & \times K: \mid \frac{P\left(\beta\left(\Phi+1 / t e_{n}\right)\right)-P(\beta \Phi)}{1 / t}- \\
& \left.-\frac{P\left(\beta\left(\Phi+1 / s e_{n}\right)\right)-P(\beta \Phi)}{1 / s} \mid \geqq \frac{1}{k}\right\} .
\end{aligned}
$$

Applying the pointwise Fubini-Jessen theorem [12] we get

$$
\begin{aligned}
& \mu\left(B_{n}\right)=\int_{R_{+} \times R \times P} \chi_{B_{n}}(s) \mu(d s) \\
& =\lim _{M \rightarrow \infty} \int \cdots \int \chi_{B_{n}}\left(\beta, \Phi^{(1)}, \Phi_{1}^{\prime}, \ldots, \Phi_{M}^{\prime}, \bar{\Phi}_{M+1 .}^{\prime} \ldots\right) \mu_{M}\left(d \beta d \Phi^{(1)} \ldots d \Phi_{M}^{\prime}\right)
\end{aligned}
$$

where $\chi_{B_{n}}$ is the caracteristic function of $B_{n}, \mu_{M}\left(d \beta \ldots d \Phi_{M}^{\prime}\right)=$ $=g\left(d \beta d \Phi^{(1)}\right) \times \prod_{m=1}^{M}\left(\frac{d \Phi_{m}^{\prime}}{2 C_{m}}\right)$ and $\bar{\Phi}^{\prime}=\sum_{m=1}^{\infty} \bar{\Phi}_{m}^{\prime} e_{m}$ is a suitable point of $K$. But as soon as $M>n$ the integral in the r.h.s. of (35) is zero because of the ordinary Fubini theorem and the well known fact that a convex function depending on one variable is differentiable except for a denumerable set of points. Hence $\mu\left(B_{n}\right)=0$ and then $\mu\left(\bigcup_{n=0}^{\infty} B_{n}\right)=0$. Let $D$ be the complement in $K$ of $\bigcup_{n=0}^{\infty} B_{n}$ then, as a consequence of the definition of $B_{n}$ and of the remark following theorem 2 , at every point of $D$ there is a unique tangent plane. Furthermore $\mu(D)=1$.

From

$$
\begin{aligned}
1=\mu(D) & =\int_{R_{+} \times R \times K} \chi_{D}\left(\beta, \Phi^{(\mathbf{1})} \Phi^{\prime}\right) g\left(d \beta d \Phi^{(1)}\right) \gamma\left(d \Phi^{\prime}\right) \\
& =\int_{K} \gamma\left(d \Phi^{\prime}\right) \int_{R_{+} \times R} \chi_{D}\left(\beta, \Phi^{(1)}, \Phi^{\prime}\right) g\left(d \beta d \Phi^{(1)}\right)
\end{aligned}
$$

and from the fact that all measures are normalized we get

$$
\int_{R_{+} \times R} \chi_{D}\left(\beta, \Phi^{(1)}, \Phi^{\prime}\right) g\left(d \beta d \Phi^{(1)}\right)=1
$$

for $\Phi^{\prime} \gamma$-almost everywhere in $K$. Then (ii) follows from the equivalence to the Lebesque measure of $g$ and from the arbitrarity of the "center" $\Phi_{0}^{\prime}$ of $K$ and of the dimensions $\left\{C_{n}\right\}$ of $K$. 
Theorems 2 and 3 specify in which sense the set of $\Phi \in \mathscr{B}$ and $\beta>0$ for which the infinite volume correlations functions exist and are unique is large.

Acknowledgements. We are greatly indebted to D. RUELLE for having suggested the problem and for many helpful discussions. We wish to thank M. L. Motchane for his kind hospitality at the IHES.

\section{References}

1. Yang, C. N., and T. D. Lee: Phys. Rev. 87, 404 (1952).

2. Ruelle, D.: Helv. Phys. Acta 36, 183 (1963).

3. Fisher, M. E.: Arch. Rat. Mech. Anal. 17, 377 (1964).

4. Griffiths, R.: J. Math. Phys. 5, 1215 (1964).

5. Dobrushin, R. L.: Teoriya Veroyatnostei i ee Primeneniya 9, 626 (1964).

6. van Hove, L.: Physica 15, 951 (1949).

7. Robinson, D., and D. Ruelle: Commun. math. Phys. 5, 288 (1967).

8. Fisher, M. E.: J. Math. Phys. 6, 1643 (1965).

9. Bourbaki, N.: Topologie générale, fascicule des resultats. § 1, 17. Paris: Hermann 1953.

10. Dunford, N., and J. Schwartz: Linear operators. V. 9.8. New York: Interscience 1958.

11. - - Linear operators. II.4.7; II.4.9. New York: Interscience 1958.

12. — - Linear operators. III.11.27. New York: Interscience 1958. 